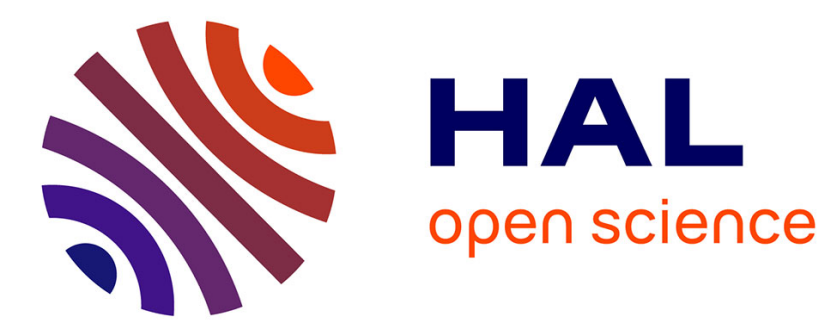

\title{
A direct comparison between gas state and atomised liquid state precursor in the deposition of functional coatings by pin corona plasma
}

P. A.F. Herbert, J. Jaroszyńska-Wolińska

\section{- To cite this version:}

P. A.F. Herbert, J. Jaroszyńska-Wolińska. A direct comparison between gas state and atomised liquid state precursor in the deposition of functional coatings by pin corona plasma. European Physical Journal: Applied Physics, 2011, 55 (1), 10.1051/epjap/2010100458 . hal-00719808

\section{HAL Id: hal-00719808 \\ https://hal.science/hal-00719808}

Submitted on 21 Jul 2012

HAL is a multi-disciplinary open access archive for the deposit and dissemination of scientific research documents, whether they are published or not. The documents may come from teaching and research institutions in France or abroad, or from public or private research centers.
L'archive ouverte pluridisciplinaire HAL, est destinée au dépôt et à la diffusion de documents scientifiques de niveau recherche, publiés ou non, émanant des établissements d'enseignement et de recherche français ou étrangers, des laboratoires publics ou privés. 


\title{
A Direct Comparison between Gas State and Atomised Liquid State Precursor in the Deposition of Functional Coatings by Pin Corona Plasma
}

\author{
P.A.F. Herbert ${ }^{1}$ and Justyna Jaroszyńska-Wolińska ${ }^{2}$ \\ ${ }^{1}$ Plasma Ireland, 22 Summerhill North, Cork, Ireland \\ ${ }^{2}$ Faculty of Civil Engineering and Architecture, Lublin University of Technology, \\ Nadbystrzycka 40, 20-618 Lublin, Poland
}

${ }^{1}$ Email: therbert@irishprecisionoptics.com

\begin{abstract}
An atmospheric pressure non-thermal equilibrium pin corona plasma jet was used to deposit polymeric coatings from monomer precursor in both vapour and liquid aerosol states to allow a direct comparison of the quality and performance of the as-deposited coatings, specifically with respect to the achievement of soft plasma polymerisation (SPP) where the coating exhibits minimal fragmentation or damage to the monomer molecule while, at the same time, being highly cross-linked. A long chain perfluorocarbon molecule was introduced into the helium plasma and coatings deposited at rates of up to $50 \mathrm{~nm} / \mathrm{min}$. XPS, FTIR, contact angle and ellipsometric measurements indicated that a controlled polymerization reaction had taken place in the case of the vapour deposited samples through the vinyl group of the monomer, with only minor fragmentation of the functional perfluoro chain. Furthermore, a high level of cross-linking was achieved and the coatings were stable to a toluene wash. In contrast, while the liquid deposition samples showed good retention of monomer molecular structure, they exhibited negligible cross-linking and were readily removed by immersion in toluene rendering them functionally useless.
\end{abstract}

\section{INTRODUCTION}

Plasma polymerization is a well established route to surface functionalisation initially developed at low pressure using gas state precursors in a technique referred to as plasma enhanced chemical vapour deposition (PECVD) in which gas precursors are bombarded with aggressive plasma species to produce fragmentation and re-arrangement of the precursor monomers. A wide variety of random fragments are created to deposit onto a substrate to produce a thin film layer containing many of the atoms present in the starting monomer. With conventional PECVD, coating functionality remains limited to simple materials such as $\mathrm{SiO}_{\mathrm{x}}, \mathrm{SiN}$ or $\mathrm{TiO}_{2}$ and complex chemistry cannot be deposited using such systems. In contrast, "soft plasma polymerization" (SPP) is the ability to plasma deposit a cross-linked solid film with a very high degree of structural retention of the starting precursor so that the deposited coating retains the molecular complexity, functionality and value of the monomer. The prospective benefits of SPP include single-step industrial coating of substrates with, for example, complex and valuable, but sensitive, bio-active macromolecules such as enzymes and nucleic acids for applications such as biosensors, lab-on-a-chip and biomedical devices and many other processes.

Around 2000, Badyal et al developed a controlled plasma polymerization process at atmospheric pressure. By combining a helium atmospheric pressure glow discharge plasma with precursor delivery as a liquid aerosol, it was possible to produce a series of thin films with soft polymerization and a high degree of retention of monomer functionality [1], [2]. This technology was further developed by Dow Corning Corporation under the brand name "APPLD” (Atmospheric Pressure Plasma Liquid Deposition) [3], [4]. Helium glow discharges were chosen as the preferred plasma option as they provide a non-thermal, homogeneous plasma that can be readily produced under ambient pressure [5]. The introduction of the liquid as an aerosol was preferred as this was thought to protect the bulk of the liquid precursor from the aggressive plasma species by encapsulating it within a droplet of several microns in diameter, thereby minimising fragmentation of the precursor monomers. The use of aerosol delivery systems, however, produces a number of complexities related to the stability of the spray, control of droplet size, generation of an even precursor distribution over wide areas, the requirement to accurately dispense low volumes of liquid at a constant rate and rapid build-up of unwanted deposits on reactor surfaces. 
In 2009, Herbert et al [6] attempted to decouple the droplet and power factors by introducing precursor as a vapour rather than aerosol into a non-thermal pin corona plasma previously used for socalled liquid deposition. The introduction of vapours rather than liquids allows for standard PECVD equipment (bubblers, mass flow controllers) to be used to generate an easily controlled, even flux of precursor onto a substrate. A standard vapour generating bubbler system was combined with a helium pin corona plasma to produce coatings using $1 \mathrm{H}, 1 \mathrm{H}, 2 \mathrm{H}, 2 \mathrm{H}$-Heptadecafluorodecyl acrylate (HDFDA) as the precursor monomer. Such coatings were shown to have the same functional chemistry retention and deposition rate as seen in 'liquid deposition' plasma coatings so that the vapour phase monomer process could be characterised as SPP and it could be deduced that control of the reaction mechanism cannot be predominantly dependent upon the presence of the monomer as an aerosol droplet but that that the key control parameter is the low plasma power coupled per unit of monomer, resulting in coatings being deposited in the power deficient regime described by Yasuda in vacuum polymerisation systems [7]. It was concluded that the specific plasma type used, the pin corona, is inherently predisposed to deliver low specific energy into the reaction zone and, hence, to SPP, even using gas precursors.

The result was confirmed by further experiments using this plasma type in which O'Neill, Herbert et al [8] showed that hexamethyldisiloxane (HMDSO) underwent SPP when injected into the plasma in the vapour phase. In the same work the liquid deposition technique failed to polymerise non-volatile polymethylhydrogen siloxane (PMHSO) atomized droplets at all flow rates. This deficiency in the ability of the pin corona liquid deposition technique to cross-link monomer precursor had been previously implied by work done by O’Neill et al [9] where PMHSO delivered as an aerosol into a pin corona system similarly could not be plasma cured except through the addition of $25-75 \%$ by volume of highly volatile tetraethylorthosilicate.

This work attempts to directly compare the performance of precursor in the conventional gas state and in the so-called liquid deposition state applied to surfaces via a pin corona plasma of low specific energy $\sim 0.1 \mathrm{~J} / \mathrm{cm}^{3}$. The precursor used was $1 \mathrm{H}, 1 \mathrm{H}, 2 \mathrm{H}-$ Perfluoro -1 - Decene (HDFD) and the resulting coatings were analysed for the two key performance parameters, namely the degree of polymerisation/cross-linking and retention of molecular structure and functionality. The precursor was chosen as it contains a polymerisable alkene group and fluorocarbon chain that can be readily characterized: $\mathrm{CH}_{2}=\mathrm{CH}-\left(\mathrm{CF}_{2}\right)_{7}-\mathrm{CF}_{3}$.

\section{EXPERIMENTAL}

A plasma jet was constructed based on the aerosol-plasma jet system described by O'Neill and O'Sullivan [10]. It consists of a dielectric head housing two tungsten needle pointed electrodes to which are applied in parallel an alternating current voltage. A space around each electrode allows a 5 $\mathrm{L} /$ minute flow of helium ballast gas to enter the device. Between the two needle electrodes is a port for insertion of a pneumatic nebuliser through which $2 \mathrm{~L} /$ minute of helium was input into the pneumatic gas feed port and a flow of $5 \mu \mathrm{L}$ /minute of liquid precursor delivered by syringe pump was input to the liquid feed port ensuring aerosol is introduced directly into the plasma region.

Alternatively, if the system is to be run in gas deposition mode, the working nebuliser is replaced in the electrode head by a 'dummy' nebuliser in which the liquid input port is blanked and a precursor plus helium carrier gas flow is fed into the gas input port. This precursor plus helium flow is the flow output by the real, working nebuliser which was configured to discharge into a heated flask the output of which flask fed directly into the dummy nebuliser. The flask was immersed in an oil bath at $200^{\circ} \mathrm{C}$ in order that the precursor with a boiling point of $146^{\circ} \mathrm{C}$ should be vaporised on emerging from the working nebuliser nozzle. The $\sim 2 \mathrm{~L} /$ minute flow rate of precursor plus helium carrier gas ensured that the time between leaving the working nebulizer and reaching the plasma region was $<1$ second reducing the time available for cooling and condensation of the precursor. No evidence of such condensation was seen.

Very Low Frequency electrical power was delivered to both electrodes at a frequency of c. $19 \mathrm{kHz}$ and a peak-to-peak voltage of c. $23 \mathrm{kV}$. The resultant helium - precursor mixture exited the system through a $75 \mathrm{~mm}$ long x $15 \mathrm{~mm}$ diameter fluoropolymer tube in which the corona plasma was struck. 
Essentially, the configuration was a conventional point-to-plane type corona configuration in which a high voltage was applied to generate plasma from the tip of vertically positioned sharp tungsten needles. Coatings were deposited onto substrates placed directly under and $2-3 \mathrm{~mm}$ from the end of the fluoropolymer tube and sitting on a ceramic block. Samples were generated in both vapour and liquid deposition modes at the following power supply set powers as a percentage of full power: $2 \%$, $5 \%, 10 \%, 20 \%$ and $40 \%$. All coating runs were 180 seconds duration. The sample was then measured for coating thickness by ellipsometry with a J.A. Woollam M2000 variable angle ellipsometer using multiple angle measurements $\left(65^{\circ}, 70^{\circ}\right.$ and $\left.75^{\circ}\right)$ over a wavelength range of 250 to $1690 \mathrm{~nm}$. In order to determine the degree of polymerization/cross-linking a toluene wash was carried out on selected samples for 5 minutes. The sample was then again characterized for coating thickness by ellipsometry.

Fourier Transform Infra-Red (FTIR) data was collected on a Bruker Vertex - 70 system. Coatings were deposited directly onto either specially thinned polished silicon substrates or $\mathrm{KBr}$ substrates and spectra were collected using 64 scans at $4 \mathrm{~cm}^{-1}$ resolution. Water contact angle measurements were obtained using the sessile drop technique using an OCA 20 video capture apparatus from Dataphysics Instruments. Drop volumes of $1.5 \mu \mathrm{l}$ were used and images were collected 30 seconds after placing the droplet on the surface. XPS analysis was performed using an Axis Ultra spectrometer (Kratos Analytical). Samples were irradiated with monochromated X-rays (Al $\mathrm{K}_{\alpha}, 1486.6 \mathrm{eV}$ ) with photoelectrons analysed from a selected area $700 \mu \mathrm{m} \times 500 \mu \mathrm{m}$, with a take-off-angle of $90^{\circ}$. Approximately $1 \times 1 \mathrm{~cm}^{2}$ pieces of the composite were taken for analysis. Each position was analysed in the survey mode (Pass Energy $160 \mathrm{eV}$ ) to determine the elements present at the surface and their relative concentrations. CasaXPS (Casa Software Ltd) data processing software was used to calculate the area under peaks representative of elements detected, which were then normalized to take into account relative sensitivity to provide relative concentrations. The elemental composition is presented as the average of three $\left(700 \times 500 \mu \mathrm{m}^{2}\right)$ areas, with $99.5 \%$ accuracy $(2 \mathrm{SD})$. FTIR, contact angle and XPS measurements were taken. -Droplet size distribution of liquid precursor nebulised through a Burgener Ari mist nebulizer was examined using the laser diffraction technique with a HELOS particle size analyser from Sympatec Gmbh. The droplet size distribution of atomised liquid and vapour phase precursor was examined using the laser diffraction technique. The applied voltage was measured using a North Star PVM-5 high voltage probe with a ratio of $1 \mathrm{~V}$ per kV directly connected to the pin electrodes. The current measurements were obtained with a Bergoz Instrumentation France, toroidal current transformer (CT-E5.0) with an output of $5 \mathrm{~V}$ per Ampere.

Electrical characterisation was by high voltage probe and toroidal current transformer.

\section{RESULTS}

Discharge powers were in the range 1.4 to $4.9 \mathrm{~W}$. The introduction of liquid droplets into the discharge at constant set power resulted in a large fall in measured discharge power from $63 \%$ to $19 \%$ depending on the setting. The laser particle size data showed that the liquid spray system introduces droplets into the plasma region with a mean particle size of less than 10 microns in all cases. Increases in precursor flow rate produced larger droplets and a wider distribution of particle sizes. Production of larger droplets with increasing flow seems intuitively reasonable due to the fixed nebulising gas flow rate of the atomizer being distributed over a larger volume per second of liquid. No droplets were observed in the vapour deposition mode confirming that the vapour system is effectively evaporating all of the introduced HDFD and that no re-condensation of the vapour occurred within the system.

Contact angle data was taken on samples run at all 5 power settings in both vapour and liquid deposition modes. It was found that in the case of the liquid deposition samples no reliable contact angle data could be obtained. Contact angles dropped from an initial value of approximately $120^{\circ}$ to approximately $30^{\circ}$ in 5 minutes for all liquid depositions. Coatings were not well cured being liquid in appearance and were readily removed by wiping. However, the vapour deposition samples showed a more robust structure. The contact angle dropped by approximately $10^{\circ}$ in the first 5 minutes after deposition and then stabilised to give the values between $107^{\circ}$ and $122^{\circ}$, typical for plasma polymerised perfluoro compounds [11]. 
Figure 1 shows the FTIR spectra of HDFD liquid deposition plasma coating at 2\% set power and vapour deposition plasma coating at $2 \%$ set power deposited on $\mathrm{KBr}$ substrates.

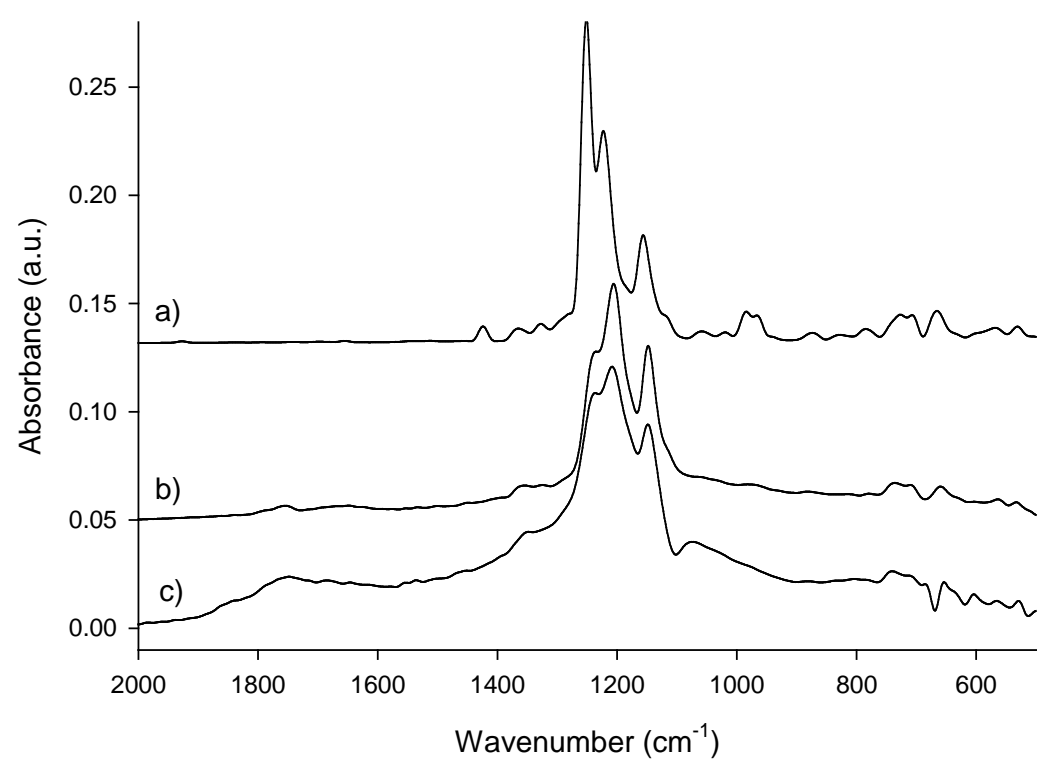

Figure 1: FTIR spectrum of a) 1H, 1H, 2H-Perfluoro-1-Decene monomer, b) liquid deposition plasma polymerisation and c) vapour deposition plasma polymerisation

The original monomer shows major peaks at 1253, 1222 and $1155 \mathrm{~cm}^{-1}$ which are attributed to the various stretching modes of the $\mathrm{CF}_{3}$ and $\mathrm{CF}_{2}$ groups of the perfluoro chain. Both the vapour and liquid deposited films also contain peaks around 1240, 1210 and $1145 \mathrm{~cm}^{-1}$, reflecting the shift to lower wavenumbers typically reported in plasma deposited fluorocarbon films and sometimes ascribed to the reduction of fluorine content in the film or to formation of a disordered and crosslinked coating [12]. As both fluorocarbon peaks are still well resolved in the coatings from both deposition processes, it can be deduced that the fluorocarbon chain has not undergone significant levels of fragmentation and degradation in either the vacuum or aerosol assisted plasma processes. Inspection of the as-deposited spectra shows loss of the monomer peaks at 1425, 967 and $990 \mathrm{~cm}^{-1}$ corresponding to loss of the $\mathrm{C}=\mathrm{C}$ bonds of the vinyl group. Both vapour and liquid deposited samples show slight peaks in the region $1700-1800 \mathrm{~cm}^{-1}$ which may suggest some $\mathrm{C}=\mathrm{O}$ formation [11], [13], [14] in agreement with the XPS analysis. The vapour deposited samples also showed peaks below $3000 \mathrm{~cm}^{-1}$ indicative of saturated alkane chemistry and confirming polymerisation has occurred through the vinyl group. These hydrocarbon features were not seen in the liquid deposition mode samples even though previous studies suggest that a similar polymerisation mechanism can occur in these aerosol assisted plasmas [1], [2], [15].

XPS analysis determined the following $\mathrm{CF}_{2}: \mathrm{CF}_{3}$ ratios for comparison with the ratio of $7: 1$ for the theoretical molecule: $2 \%$ set power liquid deposition sample $-5.8: 1 ; 2 \%$ set power vapour deposition sample $-5.6: 1$. These values are lower than expected from the starting monomer indicating that some degradation of the monomer has occurred, as is typical of all such plasma processes [6], [10], [11], [14]. However, approximately $80 \%$ of the perfluoro chain has been retained intact, which compares favourably with previously reported soft plasma polymerisation processes [16].

The $\mathrm{C}$ 1s high resolution curve fitting suggests some cross-linking in the vapour samples which is not seen in the liquid sample. A shift in binding energy position of the peak assigned to $\mathrm{CF}_{3}$ from 292.9 $\mathrm{eV}$ in the liquid deposition sample to $293.8 \mathrm{eV}$ in the vapour deposition samples suggests the presence of the $\mathrm{CF}_{3}$ species in a pendant position in the vapour samples rather than at the end of a linear chain. This may indicate scission and reformation of the molecule in the vapour phase and confirms that the fluorocarbon chain has undergone a cross-linking reaction. 
Figure 2 shows the ellipsometric data from all ten vapour and liquid deposition samples both before and after the toluene wash. For the vapour samples, the deposition rate drops off slightly with increasing plasma power which may be related to higher cross-linking producing a denser film at high power. For liquid samples, deposition rate seems erratic and does not correlate well with discharge power and may be related to the unstable contact angle data reported earlier, confirming that a stable coating was not formed using the aerosol assisted process. It was generally observed that the vapour phase coatings produced significantly higher deposition rates for most power settings. Coating stability was determined by immersing the samples in toluene and re-evaluating the coating thickness using ellipsometry. The wash test results appear to be highly significant. The vapour samples all show a minor loss of coating thickness after washing, probably due to removal of any residual poorly polymerised material. However, the cross-linking detected in the high resolution curve fitting of the XPS spectra appears to be sufficient to stabilise the film and provides for significant wash resistance. In contrast, in the liquid samples it appears almost no cross-linking has taken place so that wash resistance is non-existent and the entire coating is removed in all cases.

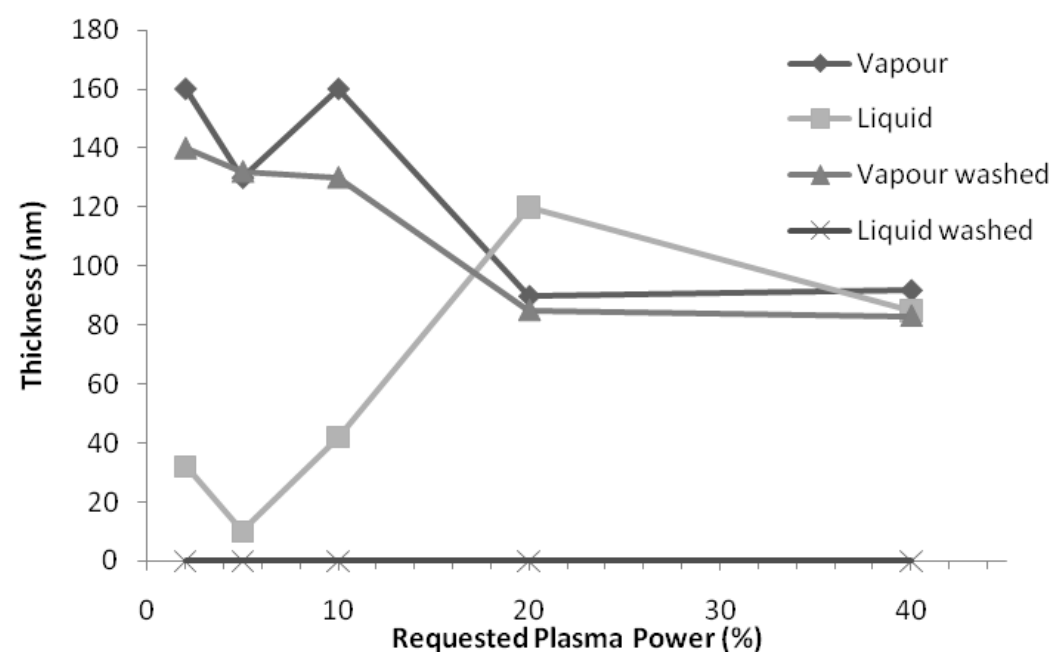

Figure 2: Ellipsometric coating thickness data from vapour and liquid deposited samples at 5 set powers before and after toluene wash

\section{DISCUSSION}

A low power, non-isothermal equilibrium, atmospheric pressure corona plasma has been generated from a single electrode with pin geometry and used to deposit plasma polymerized coatings from HDFD monomer precursor in both the gas state and in the liquid aerosol state.

The large reduction seen in discharge power on introduction of the aerosol is probably due to the nebulised droplets acting as a sink for free electrons. The fast electrons will be preferentially lost due to their increased speed and, thus, collision rate and because they are able to better overcome the negative potential of the plasma sheath (the floating potential) set up by the droplet to ensure that the droplet is bombarded by equal fluxes of slow ions and fast electrons thus maintaining overall charge neutrality. Essentially, the liquid droplets act as a cold boundary surface to the plasma sinking charged species and imposing an overall cooling effect on the plasma. They are likely to change the Electron Energy Distribution Function by reducing both the mean electron energy and the high energy end or tail of the distribution. This reduction in electron density reduces the number of free electrons available to sink power directly resulting in the decrease in plasma power seen.

The vapour deposited samples showed that it was possible to deposit cured polymeric coatings which substantially retained the chemical structure of the precursor monomer so that the process could be characterised as SPP. The coating was hydrophobic and was put down at reasonable deposition rates. Analysis of the coatings clearly shows that the precursor has undergone a controlled polymerization through the vinyl component of the molecule with low fragmentation of the functional fluorocarbon chemistry of the monomer. Some levels of, but not complete, oxidation of the coatings were evidenced by carbonyl and water peaks detected by FTIR and oxygen by XPS, as might be expected 
in this open perimeter system with the substrate exposed to ambient air. The absence of complete oxidation is consistent with the low specific power levels generated by this plasma type. The resultant coatings, however, produced XPS and FTIR spectra fully comparable to those produced by pulsed vacuum plasma or by liquid aerosol plasma processing. Furthermore, wash tests and FTIR and XPS data showed clear evidence of a high level of cross-linking, an essential attribute of a viable functional coating.

In contrast, although the liquid deposition samples showed good chemistry retention, the XPS data suggested that cross-linking within the deposited coatings was negligible and the resultant wash tests rendered the coatings functionally useless, even at plasma power levels equal to those of vapour deposition processes. Difficulty in obtaining adequate levels of polymerisation of liquid state atomised precursors have been seen in previous work [9], [17].

It seems plausible to associate both deposition rate and the extent of cross-linking of the deposited coating with the degree of monomer interaction with the plasma at the molecular level. It is selfevident that monomer molecules in the vapour phase will be fully exposed to interaction with plasma species from entry into the plasma region, throughout their flight and on the substrate surface. In contrast, most monomer in the liquid state will be shielded from the plasma, certainly during flight and probably to a significant extent on the substrate surface when the droplet wets out to spread across an area, leaving only the surface available to interact directly with the plasma. Vapour phase monomer molecules will, therefore, have a much higher probability of engaging in plasma induced chemical reactions than liquid phase molecules so that the vapour phase reaction rate will be substantially higher generating more cross-linking and thicker coatings at low plasma power. The greatly enhanced deposition rate and wash resistance of the vapour derived coatings clearly support this assumption.

\section{CONCLUSIONS}

This paper details what appears to be the first reported direct comparison of the performance of coatings targeting soft plasma polymerization (SPP) from both gas and liquid aerosol state precursors using a cool, atmospheric pressure, highly non-isothermal equilibrium pin corona discharge with a low specific energy $\sim 0.1 \mathrm{~J} / \mathrm{cm}^{3}$.

The work confirmed that, with this type of corona discharge, largely damage-free polymerization of monomer molecules to deposit a functional coating can be readily achieved with precursor in the conventional gas state, i.e. operating in standard PECVD mode. This suggests that the use of precursor in the liquid state as nebulised droplets is not required to achieve SPP, as has been suggested elsewhere [1], [2], [3], [18], [19], [20], [21]. Vapour deposition showed high retention of monomer molecular structure in the coating combined with excellent cross-linking and durability, the two key SPP parameters of merit.

In contrast, the same process but using precursor as liquid aerosol in the so-called liquid deposition mode was unable to achieve a comparable coating. Good retention of monomer molecular structure was seen in the liquid coatings, but cross-linking within the coating was negligible thereby rendering the deposits functionally useless. 


\section{REFERENCES}

[1] Ward, L.J.; Schofield, W.C.E.; Badyal, J.P.S.; Goodwin, A.J.; Merlin, P.J. Chem. Mater, 2003, 15, 1466

[2] Ward, L.J.; Schofield, W.C.E.; Badyal, J.P.S.; Goodwin, A.J.; Merlin, P.J. Langmuir, 2003, 19(6), 2110

[3] O’Hare, L.-A.; O’Neill, L.; Goodwin, A. J. Surf. Interface. Anal., 2006, 38 (11), 1519

[4] Albaugh, J.D.; O’Sullivan, C.; O’Neill, L. Surf. Coat. Technol., 2008, 203, 844

[5] Massines, F. Gouda, G. J. Phys. D: Appl. Phys, 1998, 31, 3411

[6] Herbert, P.A.F., O’Neill, L., Jaroszynska-Wolinska, J., Chem. Mater., 2009, 21, 4401-4407

[7] Yasuda, H. Journal of Polymer Science: Macromolecule Reviews, 1981, 16, 199

[8] O’Neill, L., Herbert, P.A.F., Stallard, C., Dowling, D.P, Plasma Process. Polym., 2010, 7, 43-50

[9] L. O’Neill et al, "Plasma Polymerised Primers - Improved Adhesion through Polymer Coatings”, Society of Vacuum Coaters, $50^{\text {th }}$ Annual Technical Conference Proceedings, 2007

[10] O’Neill, L.; O’Sullivan, C. Chem Vap. Dep., 2009, 1-3, 21

[11] Coulson, S. R., Woodward, I. S., Badyal, J. P. S., Brewer, S.A., Willis, C., Langmuir, 2000, 16, $6287-6293$

[12] Fanelli F., Fracassi, F, d’Agostino R., Plasma Process Polymer., 2007, 4, S430-434

[13] Fanelli, F. Et al, Plasma Process. Polym., 2009, 6, S503-S507

[14] Laguardia, L., Ricci, D., Vassallo, E., Cremona, A., Mesto, E., Grezzi, F., Dellera, F., Macromol. Symp., 2007, 247, 295-302

[15] Beck, A.J.; Candan, S., Short, R.D.; Goodyear, A.; Braithwaite, N.S.J. J. Phys. Chem. B, 2001, 105,5730

[16] Coulson, S. R., Woodward, I. S., Badyal, J. P. S., Brewer, S. A., Willis, C., Chem. Mater., 2000, 12,2031

[17] Herbert, P. A. F., "Investigation of droplet behaviour in the deposition of functional coatings using atomised liquid precursors in cool, atmospheric pressure plasma", Hakone XI Proceedings of $11^{\text {th }}$ International Symposium on High Pressure, Low Temperature Plasma Chemistry, Oleron Island, France, 7-11 Sep 2008, pp.488-492

[18] Heyse, P.; Dams, R.; Paulussen, S.; Houthoofd, K.; Janssen, K.; Jacobs, P.A.; Sels, B. F. Plasma Process. Polym., 2007, 2, 145

[19] Tatoulian, M. ; Arefi-Khonsari, F. Plasma Process. Polym., 2007, 4, 360

[20] Beck, A.J.; Short, R.D.; Matthews, A. Surf. Coat. Technol., 2008, 203 (5-7), 822-825

[21] Hynes, A. et al, "Generation and Control of Wide-area Homogeneous Atmospheric Pressure Glow Discharges for Industrial Coating Applications”, Hakone IX International Symposium on High Pressure, Low Temperature Plasma Chemistry, Padova, Italy, 2004, 8O-03 